\title{
E2F1 inhibits circulating cholesterol clearance by regulating Pcsk9 expression in the liver
}

\author{
Qiuwen Lai, ${ }^{1}$ Albert Giralt, ${ }^{1,2}$ Cédric Le May, ${ }^{3}$ Lianjun Zhang, ${ }^{4}$ Bertrand Cariou, ${ }^{3}$ \\ Pierre-Damien Denechaud, ${ }^{1,2}$ and Lluis Fajas ${ }^{1,2}$ \\ ${ }^{1}$ Department of Physiology, ${ }^{2}$ Center for Integrative Genomics, University of Lausanne, Lausanne, Switzerland. \\ 3|'Institut du Thorax, Inserm UMR1087 - CNRS UMR6291, UNIV Nantes, Nantes, France. ${ }^{4}$ Ludwig Cancer Research and \\ Department of Oncology, University of Lausanne, Epalinges, Switzerland.
}

\begin{abstract}
Cholesterol accumulation in the liver is an early event in nonalcoholic fatty liver disease (NAFLD). Here, we demonstrate that E2F1 plays a crucial role in maintaining cellular cholesterol homeostasis by regulating cholesterol uptake via proprotein convertase subtilisin/kexin 9 (PCSK9), an enzyme that promotes low-density lipoprotein receptor (LDLR) degradation upon activation. E2 $\mathrm{fr}^{-/-}$mice display reduced total plasma cholesterol levels and increased cholesterol content in the liver. In this study, we show that E2f1 deletion in cellular and mouse models leads to a marked decrease in Pcsk 9 expression and an increase in LDLR expression. In addition to the upregulation of LDLR, we report that $\mathrm{E} \mathrm{f1}^{-1-}$ hepatocytes exhibit increased LDL uptake. ChIP-Seq and PCSK9 promoter reporter experiments confirmed that E2F1 binds to and transactivates the PCSK9 promoter. Interestingly, E2 $\mathrm{f1}^{-/-}$mice fed a high-cholesterol diet (HCD) display a fatty liver phenotype and liver fibrosis, which is reversed by reexpression of PCSK9 in the liver. Collectively, these data indicate that E2F1 regulates cholesterol uptake and that the loss of E2F1 leads to abnormal cholesterol accumulation in the liver and the development of fibrosis in response to an HCD.
\end{abstract}

Authorship note: PDD and LF contributed equally to this work.

Conflict of interest: The authors have declared that no conflict of interest exists.

Submitted: July 25, 2016 Accepted: April 13, 2017 Published: May 18, 2017

\section{Reference information:} JCI Insight. 2017;2(10):e89729. https://doi.org/10.1172/jici. insight.89729.

\section{Introduction}

There are 8 E2F genes (E2F1-8) in mammals, which can be widely classified based on their transcriptional properties, cell cycle functions, and interaction partners (1). Among the E2Fs, E2F1 is the best-studied transcription factor controlling the G1/S transition during the cell cycle. The activity of E2F1 is dependent on its binding partners: dimerization protein (DP) and the retinoblastoma (RB) family of proteins (2). Upon cell cycle entry, cyclin-dependent kinases 4/6 (cdks 4/6) phosphorylate RB, leading to release of the E2F1$\mathrm{RB}$ repressor complex (3), resulting in activation of E2F1-mediated transcriptional regulation of target genes, such as thymidine kinase $(T k)$, dihydrofolate reductase $(D h f r)$, cyclin $\mathrm{D}$, and cyclin $\mathrm{E}$. In addition to cell cycle control, E2F1 also participates in the control of oxidative, glucose, and lipid metabolism (4-6). Moreover, the E2f1 ${ }^{-/-}$mouse model exhibits decreased plasma total cholesterol levels, suggesting that E2F1 may also be implicated in regulating cholesterol homeostasis (7). Here, we elucidate a mechanism explaining how E2F1 participates in controlling sterol balance.

Cholesterol is an essential building block for the cell membrane and is a precursor of metabolites such as bile acids (BA), steroid hormones, and oxysterols, which have important biological functions in physiology. Cholesterol is a highly insoluble molecule that must be transported in the form of lipoprotein particles. There are 4 main types of circulating lipoproteins: chylomicron (CM), very low-density lipoprotein (VLDL), low-density lipoprotein (LDL), and high-density lipoprotein (HDL). Perturbation of the cholesterol balance leading to high plasma LDL levels and low HDL levels is a risk factor for cardiovascular diseases (CVDs). Thus, interventions aimed at decreasing plasma LDL levels have become an efficient strategy to protect against CVDs.

The liver is one of the primary organs that maintains whole-body cholesterol homeostasis (8). In the liver, cholesterol is packaged in VLDL and then enters the circulation, where VLDL undergoes lipolysis to form LDL. The uptake of LDL and other ApoE/ApoB lipoproteins is mediated by low-density lipoprotein receptor (LDLR). LDL binds to LDLR and is endocytosed into cells (9). LDLR levels are 
A

Plasma cholesterol

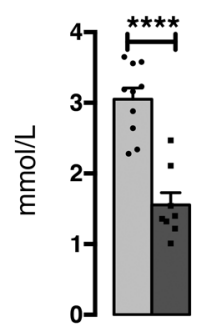

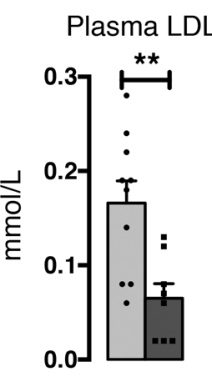

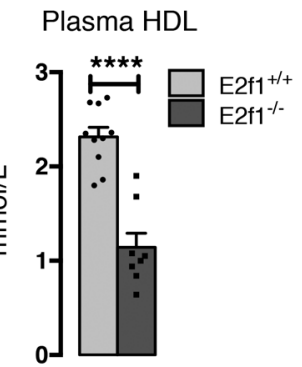

B

Total cholesterol in feces

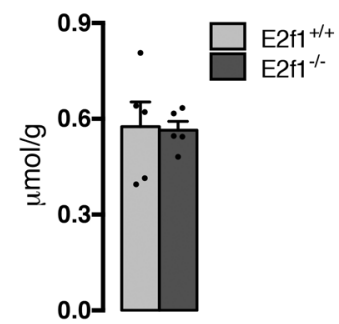

C Fecal total BA

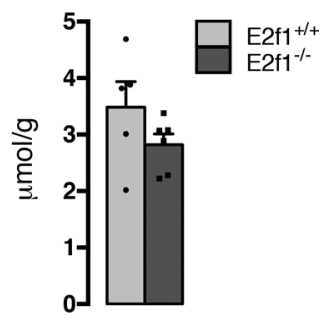

D

Cholesterol content in tissues

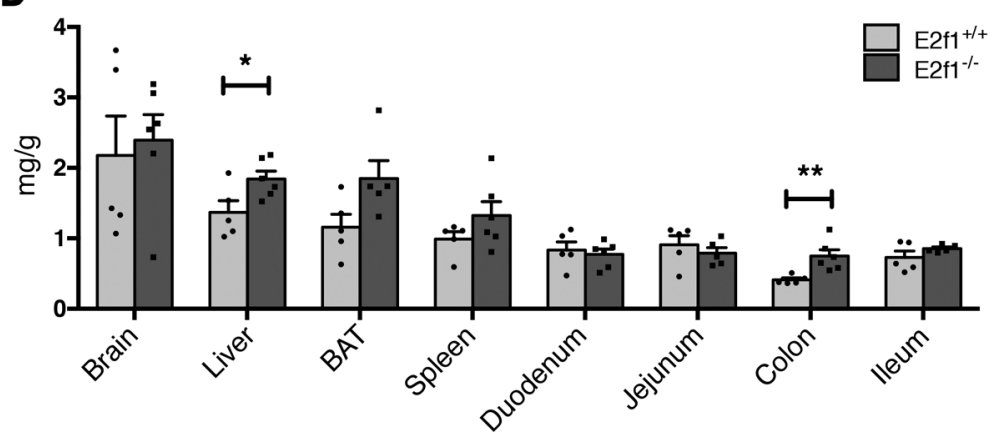

Figure 1. Decreased total plasma cholesterol levels and increased cholesterol content in the liver and colon tissues of E2f1 ${ }^{-I-}$ mice. (A) Plasma lipid profiles of $E 2 \mathrm{f1}^{+/+}$and $E 2 \mathrm{fr}^{-I_{-}}$mice fed a normal chow diet. $n=8-10$ mice per group. (B) Quantification of total free cholesterol levels in feces collected from individual mice using commercial kits. $n=5$ mice per group. (C) Quantification of total bile acid (BA) levels in feces collected from individual mice using

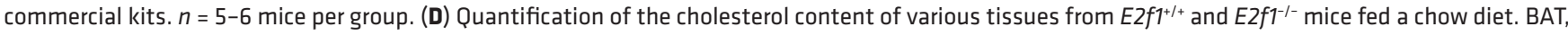
Brown adipose tissue. $n=5-6$ mice per group. All data are presented as the mean \pm SEM. Differences between $E 2 f^{1^{/+}}$and $E 2 \mathrm{f1}^{-l_{-}^{-}}$were determined by 2-tailed unpaired $t$ test. ${ }^{*} P<0.05,{ }^{* *} P<0.01,{ }^{* * *} P<0.0001$.

crucial for maintaining the optimal cellular cholesterol balance (10). LDLR levels are transcriptionally controlled by sterol regulatory element-binding protein 2 (SREBP2) and posttranscriptionally controlled by proprotein convertase subtilisin/kexin 9 (PCSK9), an enzyme that binds to LDLR and promotes its degradation in the lysosomal pathway. Inhibition of PCSK9 prevents LDLR degradation, resulting in increased availability of LDLR to facilitate LDL clearance. Hence, PCSK9 inhibitors have been developed as novel drugs for CVDs (11).

In this study, we elucidate a new mechanism upstream of PCSK9-mediated LDLR degradation. We show that E2F1 acts as a positive regulator of PCSK9 to control cholesterol uptake. In the absence of E2F1-induced PCSK9 regulation, there is an increase in LDLR-mediated uptake and overaccumulation of cholesterol in cells. E2f1-/- mice fed a high-cholesterol diet (HCD) display increased $\alpha$-collagen and $\alpha$-sma gene expression, which is typically observed during liver fibrosis development. This phenotype is reversed by reexpression of PCSK9 in liver by adenoviral strategy. Taken together, our results show that, in addition to SREBP2, E2F1 acts to control LDLR via PCSK9 in response to sterol signals to prevent overaccumulation of sterols in the cell, particularly during high-cholesterol dietary intake.

\section{Results}

E2f1-1- mice exhibit decreased plasma cholesterol levels and increased cholesterol content in the liver and colon tissues. Previous results from our laboratory indicated that E2F1 is a major regulator of lipid metabolism (5). By analyzing the plasma lipid profile, we found that $E 2 F 1-\mathrm{KO}$ mice $\left(E 2 f 1^{-/}\right)$display lower plasma total cholesterol, HDL-cholesterol, and LDL-cholesterol (LDL-C) levels compared with WT mice $\left(E 2 f 1^{+/+}\right)$fed a normal chow diet (Figure 1A). These results were confirmed via FPLC separation of lipoproteins (Supplemental Figure 1; supplemental material available online with this article; https://doi.org/10.1172/ jci.insight.89729DS1) and suggested that deletion of E2f1 disrupts cholesterol homeostasis.

We quantified the cholesterol and BA content in the feces to elucidate whether the observed reduction in circulating cholesterol levels was secondary to increased cholesterol excretion through the biliary and/or the transintestinal pathways. No differences were observed between the $E 2 f 1^{+/+}$and $E 2 f 1^{-/-}$mice (Figure 1, B 
Table 1. List of cholesterol-related genes enriched in E2F1 ChIP-seq analysis of primary culture of hepatocytes Ad-E2F1

\begin{tabular}{|c|c|c|}
\hline ENSEMBL_GENE_ID & GENE_ID & Gene name \\
\hline ENSMUSG00000018167 & Stard3 & START domain containing 3 \\
\hline ENSMUSG00000018861 & Fdxr & ferredoxin reductase \\
\hline ENSMUSG00000020538 & Srebf1-c & sterol regulatory element binding transcription factor $1-c$ \\
\hline ENSMUSC00000020919 & Stat5b & signal transducer and activator of transcription 5B \\
\hline ENSMUSC00000021259 & Сур46а1 & cytochrome $\mathrm{P} 450$, family 46 , subfamily a, polypeptide 1 \\
\hline ENSMUSG00000021273 & Fdft1 & farnesyl diphosphate farnesyl transferase 1 \\
\hline ENSMUSG00000021594 & Srd5a1 & steroid 5 alpha-reductase 1 \\
\hline ENSMUSG00000023832 & Acat2 & acetyl-Coenzyme $A$ acetyltransferase 2 \\
\hline ENSMUSC00000023921 & Mut & methylmalonyl-Coenzyme A mutase \\
\hline ENSMUSC00000024087 & Cyp1b1 & cytochrome $\mathrm{P} 450$, family 1 , subfamily b, polypeptide 1 \\
\hline ENSMUSG00000024687 & Osbp & oxysterol binding protein \\
\hline ENSMUSG00000024799 & Tm7sf2 & transmembrane 7 superfamily member 2 \\
\hline ENSMUSC00000026675 & Hsd17b7 & hydroxysteroid (17-beta) dehydrogenase 7 \\
\hline ENSMUSG00000030670 & Сур2r1 & cytochrome $\mathrm{P} 450$, family 2 , subfamily r, polypeptide 1 \\
\hline ENSMUSC00000031393 & Mecp2 & methyl $\mathrm{CpC}$ binding protein 2 \\
\hline ENSMUSG00000031604 & Sc4mol & sterol-C4-methyl oxidase-like \\
\hline ENSMUSC00000031708 & Tecr & Trans-2,3-Enoyl-CoA Reductase \\
\hline ENSMUSC00000032245 & $C \ln 6$ & ceroid-lipofuscinosis, neuronal 6 \\
\hline ENSMUSC00000037295 & Ldlrap1 & low density lipoprotein receptor adaptor protein 1 \\
\hline ENSMUSG00000039050 & Osbpl2 & oxysterol binding protein-like 2 \\
\hline ENSMUSG00000039529 & Atp8b1 & ATPase, class I, type 8B, member 1 \\
\hline ENSMUSG00000040374 & Pxmp3 & peroxisomal membrane protein 3 \\
\hline ENSMUSC00000041939 & Mvk & mevalonate kinase; similar to mevalonate kinase \\
\hline ENSMUSC00000044252 & Osbpl1a & oxysterol binding protein-like $1 \mathrm{~A}$ \\
\hline ENSMUSG00000044254 & Pcsk9 & proprotein convertase subtilisin/kexin type 9 \\
\hline ENSMUSC00000045294 & Insig1 & insulin induced gene 1 \\
\hline ENSMUSC00000046873 & Mbtps2 & membrane-bound transcription factor peptidase, site 2 \\
\hline ENSMUSG00000058454 & Dher7 & 7-dehydrocholesterol reductase \\
\hline ENSMUSG00000059743 & Fdps & Farnesyl Diphosphate Synthase \\
\hline
\end{tabular}

and C). We next investigated whether the cholesterol content in the tissues was altered in these mice. Interestingly, the $E 2 \mathrm{fl}^{-/-}$mice exhibited consistently higher levels of cholesterol in the liver and colon compared with $E 2 \mathrm{fl}^{+/+}$mice (Figure 1D). No major differences in cholesterol content were detected in the other tissues tested. These findings suggested that the reduction of plasma cholesterol levels observed in the E2f1-/- mice was potentially due to increased cholesterol uptake.

E2F1 participates in the transcriptional regulation of genes involved in cholesterol metabolism. As a transcription factor, E2F1 controls the expression of its target genes. We analyzed our previously published E2F1 ChIP high-throughput DNA sequencing (ChIP-seq) data from primary cultures of mouse hepatocytes infected with adenovirus $E 2 F 1$ (5) to identify potential E2F1 targets that regulate cholesterol homeostasis. We found that E2F1 binds to the regulatory regions of several cholesterol-related genes (Table 1), including mevalonate kinase, 7 dehydrocholesterol reductase, and PCSK9 (Supplemental Figure 2). PCSK9 is a promising target because it is a key regulator of cholesterol homeostasis that controls LDL-C uptake (12). ChIP-quantitative PCR (ChIP-qPCR) experiments in primary Ad-E2F1-infected hepatocytes and in a human hepatocellular liver carcinoma cell line (HepG2) confirmed our ChIP-seq data and showed that endogenous E2F1 specifically binds to the PCSK9 promoter (Figure 2A and Supplemental Figure 3). In addition, the functional occupancy of E2F1 on the human PCSK9 promoter was assessed through Renilla-based reporter analyses. Consistently, transient transfections revealed that $\mathrm{E} 2 \mathrm{~F} 1$ was able to transactivate the $P C S K 9$ promoter (Figure $2 \mathrm{~B}$ ). To assess the hepatocyte specificity of this regulation, we performed ChIP and gene expression analysis in mouse embryonic fibroblasts (MEF), which express very low levels of Pcsk9. We did not observed any variation in Pcsk9 mRNA between $\mathrm{E}_{2} \mathrm{fl}^{+/+}$and $\mathrm{E} 2 \mathrm{fl}^{-/-} \mathrm{MEFs}$ or E2F1 binding to Pcsk9 promoter (Supplemental Figure 4, 
A ChIP Pcsk9 promoter

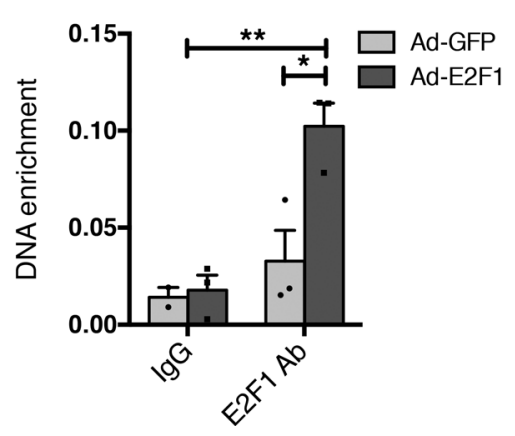

B Pcsk9 promoter reporter assay

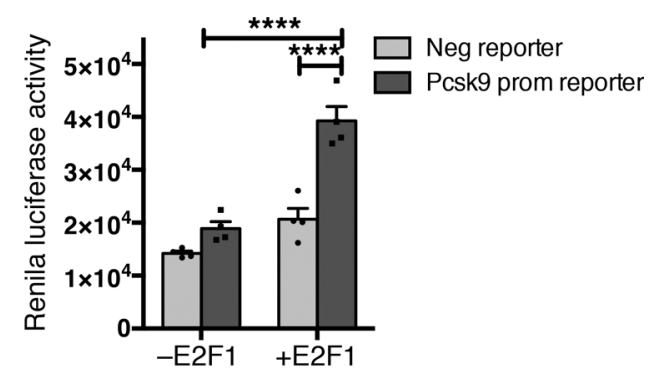

Figure 2. E2F1 binds and transactivates Pcsk9 promoter activity. (A) E2F1 ChIP on hepatocytes expressing Ad-E2F1 demonstrated E2F1 binding to Pcsk9 promoter. All data are presented as the mean \pm SEM from 2-3 independent experiments. Differences were determined by 2 -way ANOVA. ${ }^{*} P<0.05$, ${ }^{*} P<0.01$. (B) Human $P$ CSK9 promoter reporter activity in HepG2 cells transfected with E2F1 or the empty plasmid. Experiences were performed 3 times with similar results. A representative experiment performed in quadruplicate is shown. All data are presented as the mean \pm SEM. Differences were determined by 2 -way ANOVA. ${ }^{* * *} P<0.0001$.

A-C). Collectively, our results indicate that E2F1 binds and transactivates the Pcsk9 promoter in the liver.

E2F1 is a positive regulator of Pcsk9.

We decided to focus on the liver and hepatocytes to further characterize the effects of E2F1 on Pcsk9 transcription because the liver is the central organ for cholesterol homeostasis. The first interesting observation was that Pcsk9 expression was regulated by E2F1. In agreement with previous studies (13), hepatic Pcsk9 mRNA expression was increased in the livers of mice in the fed state (Figure 3A). However, this induction was significantly reduced in the livers of $E 2 f 1^{-/-}$mice compared with $E 2 f 1^{+/+}$mice. By contrast, E2f1

deficiency did not alter Pcsk9 expression in the fasting state (Figure 3A). PCSK9 protein expression in hepatic samples derived from $E 2 \mathrm{fl}^{-{ }^{--}}$mice was also reduced in the fed state (Figure $3 \mathrm{~B}$ ). In accordance with the typical role of PCSK9 in inducing LDLR degradation, we decided to investigate the LDLR protein in the livers of $E 2 \mathrm{fl}^{-/-}$compared with $E 2 f 1^{+/+}$mice. Probably because of the nutritional regulation of $P c s k 9$, we were not able to see major difference in LDLR protein level between $E 2 f 1^{+/+}$and $E 2 f 1^{-/-}$mice, as assessed by Western blot (Figure 3B). For this reason, we switched to a cellular model to explore the molecular mechanisms involved in this regulation in a primary culture of hepatocytes. Basal Pcsk9 mRNA was

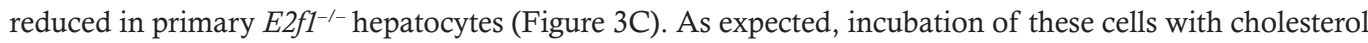
decreased Pcsk9 expression (Figure 3C). This reduction was even more pronounced in $E 2 \mathrm{fl}^{-{ }^{--}}$hepatocytes at the mRNA and protein levels (Figure 3, C and D). Consistently, LDLR protein expression, analyzed through immunofluorescence, was increased in E2f1-1- cells (Figure 3E). Strikingly, and possibly as a result of increased LDLR expression and activity, the cholesterol content was increased in $E 2 \mathrm{fl}^{-/-}$hepatocytes (Figure $3 \mathrm{~F}$ ). We investigated the rate of cholesterol biosynthesis in the cells to determine whether the higher cholesterol content of $E 2 \mathrm{fl}^{-{ }^{-}-}$hepatocytes was due to an increase in de novo cholesterol synthesis. E2f1-/ hepatocytes exhibited a lower rate of cholesterol biosynthesis compared with $E 2 f 1^{+/+}$hepatocytes (Figure 3G). At the gene-expression level, E2f1-/- hepatocytes exhibited a decrease in Srebp2, Hmgcs1, Hmgcr, and $F d f t 1$ gene expression but not $M v d, M v k$, and $D h c r 7$ compared with $E 2 f 1^{+/+}$hepatocytes (Supplemental Figure 5). We also found no differences in BA synthesis gene expression but a decrease in Abca1, Abcg1, Abcg5, and $A b c g 8$ gene expression, transporters that are important for cholesterol efflux through BA (Supplemental Figure 5) (14). These results confirmed that the high cholesterol levels observed in E2f1-/- cells are due to an increase in sterol uptake and not endogenous biosynthesis.

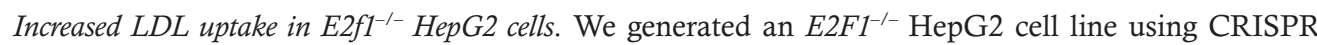
technology in which the E2F1 protein was successfully removed to validate our results obtained from mouse hepatocytes in a human cell model (Figure 4B). Interestingly, the expression of both PCSK9 mRNA and protein was decreased in $E 2 \mathrm{FI}^{-1-}$ cells compared with the control cells (Figure 4, A and B). Consistent with the decrease in PCSK9 expression, LDLR protein expression was increased in $E 2 \mathrm{FI}^{-/-}$cells (Figure 4D). The augmented LDLR expression contributed to an increased LDL uptake in $E 2 \mathrm{FI}^{-1-}$ cells (Figure $4 \mathrm{C})$. Because cholesterol accumulation inhibits cholesterol biosynthesis, $E 2 \mathrm{Fl}^{-1-}$ cells consistently showed a decrease in the capacity to synthesize cholesterol (Figure 4E). Taken together, these results supported the hypothesis that E2F1 acts as a positive regulator of hepatic PCSK9 in human and mouse hepatocytes. The reduction of PCSK9 expression in E2F1-deficient cells or tissues facilitates LDL-C uptake through LDLR upregulation, which contributes to the accumulation of cellular cholesterol and the decrease in endogenous cholesterol biosynthesis (Figure $4 \mathrm{~F}$ ).

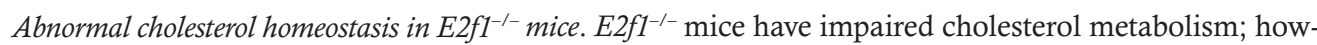
ever, they do not show a pathological phenotype on a regular chow diet. To further investigate the participation of E2F1 in the regulation of cholesterol metabolism in vivo, we challenged the mice with 5 weeks of 

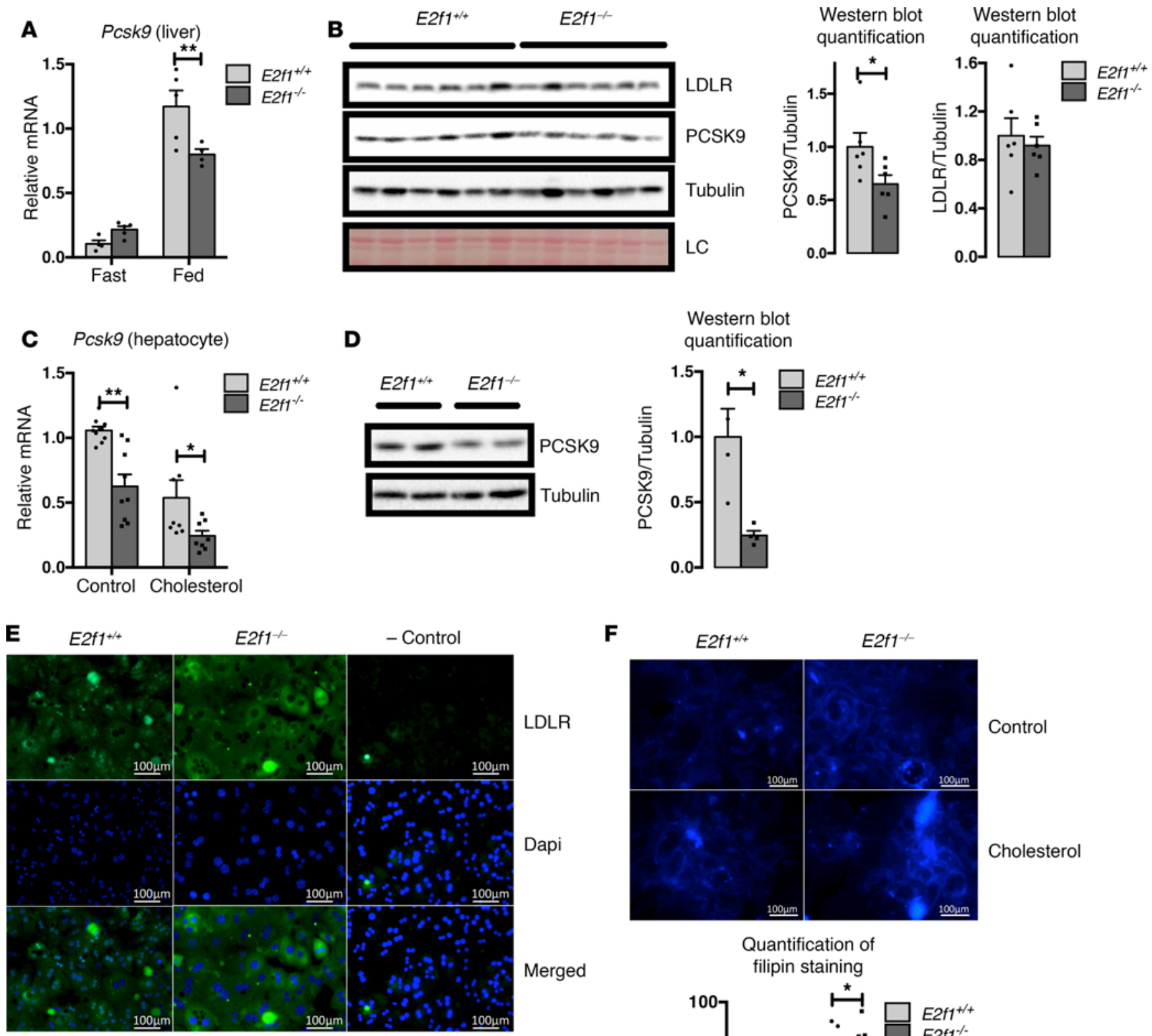

Quantification of

filipin staining

G Cholesterol biosynthesis (hepatocytes)
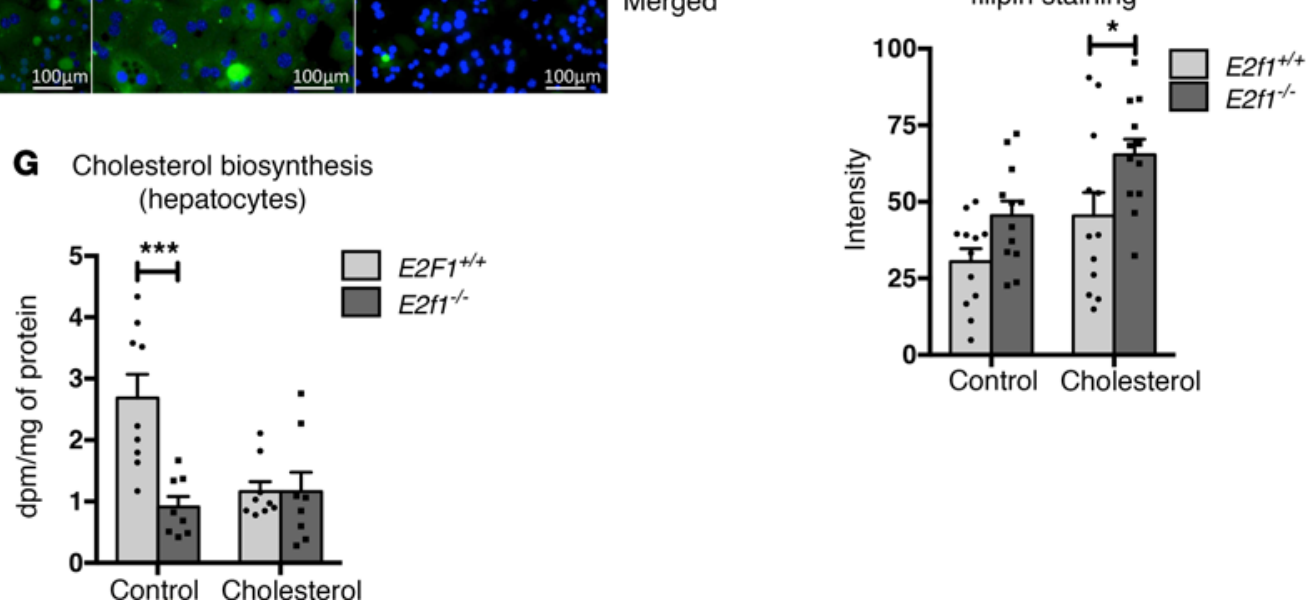

Figure 3. Loss of E2f1 decreases Pcsk9 expression and increases LDLR protein expression in hepatocytes. (A) Relative expression of Pcsk9 mRNA in livers from $E 2 \mathrm{f1}^{+/+}$and $E 2 \mathrm{f1}^{-/-}$mice that were fed a normal chow diet or fasted overnight. (B) Western blot of PCSK9 and LDLR in the livers of $E 2 \mathrm{ff}^{+/+}$ and $E 2 \mathrm{f1}^{-/-}$mice. Ponceau staining of the membrane are shown as a loading control (LC). Normalizations of the quantified values are represented. Differences between $E 2 \mathrm{f1}^{+/+}$and $E 2 \mathrm{f1}^{-/-}$were determined by 2 -tailed unpaired $t$ test. ${ }^{*} P<0.05$. (C) Relative expression of Pcsk9 mRNA in primary $E 2 \mathrm{f1}^{+/+}$and $E 2 \mathrm{fr}^{-/-}$mouse hepatocytes treated with medium supplemented with DMSO (control, ctrl) or $0.065 \mathrm{mg} / \mathrm{ml}$ cholesterol (chol). Three independent experiments in triplicate. (D) Representative images of PCSK9 Western blots from primary E2f1+/+ and E2f1 $1^{-/-}$mouse hepatocytes in medium with cholesterol. Tubulin was used as a loading control $(n=2)$. Normalization of the quantified values is represented. Differences between $E 2 f 7^{+/+}$and 
$E 2 f 1^{-/-}$were determined by 2-tailed unpaired $t$ test. ${ }^{*} P<0.05$. (E) Representative images of LDLR immunostaining in primary $E 2 f 1^{+/+}$and $E 2 f 1^{-/-}$mouse hepatocytes $(n=3)$. Experiments were independently repeated 3 times. LDLR is stained in green; nuclei are stained in blue. Scale bars: $100 \mu m$. (F) Filipin staining of $E 2 f 1^{+/+}$and $E 2 f 1^{-/-}$mouse hepatocytes $(n=3)$. Quantification of the intensity of filipin staining are represented. Scale bars: $100 \mu$ m. (G) Quantification of the rate of cholesterol biosynthesis in $E 2 f 1^{+/+}$and $E 2 f 1^{-/-}$mouse hepatocytes treated with medium supplemented with DMSO (ctrl) or $0.065 \mathrm{mg} / \mathrm{ml}$ cholesterol (chol). All data are presented as the mean \pm SEM. Differences between $E 2 f 1^{+/+}$and $E 2 f 1^{-/-}$were determined by $2-$ way ANOVA unless indicated otherwise. ${ }^{*} P<0.05,{ }^{*} P<0.01,{ }^{* *} P<0.005$

feeding with an HCD. As previously described (Figure 1), E2f1 $1^{-/-}$mice showed reduced plasma cholesterol levels compared with $E 2 \mathrm{fl}^{+/+}$mice before the change of diet (Figure $5 \mathrm{~A}$ ). The HCD led to a time-dependent increase in plasma cholesterol levels in both genotypes (Figure 5A). At the end of the 5-week HCD treatment, we did not observe any difference in plasma total, HDL-, or LDL-C levels between E2f1 ${ }^{+/+}$ and $E 2 f^{-1-}$ mice (Figure 5, A-C). In contrast, we observed major morphological differences in the livers of these mice. Surprisingly, analysis of H\&E staining of liver sections of E2 $\mathrm{f1}^{-/-}$mice revealed morphological changes of the hepatocytes that suggested nonalcoholic fatty liver disease (NAFLD) (Figure 5D). Moreover, the neutral lipid content, as determined by Oil Red O staining, was also increased in the livers of the $E 2 f 1^{-1-}$ mice compared with the $E 2 f 1^{+/+}$mice (Figure 5D), highlighting that $E 2 f 1$ deletion led to an increase in lipid accumulation in this tissue (Figure 5E). Free-cholesterol and filipin staining, a marker of the cholesterol content, was also stronger in $E 2 \mathrm{fl}^{-/-}$livers (Figure 5E and Supplemental Figure 6). Strikingly, the expression of Pcsk9 at both the mRNA and protein levels was diminished in the livers of $E 2 f^{1^{-1-}}$ mice compared with $E 2 \mathrm{fl}^{+/+}$mice fed the HCD (Figure 5, F and G). This result was consistent with the increased LDLR expression observed in the livers of the E2f1-/- mice (Figure 5, H and I). In addition, we found that the expression of $C d 36$ - a member of the scavenger receptor class $\mathrm{B}$, which participates in the uptake of fatty acids - was increased in the liver of $E 2 \mathrm{fl}^{-{ }^{--}}$mice on HCD compared with control mice and could also explain the observed increase in intracellular lipids in the liver of E2f1 ${ }^{-/-}$mice (Figure $5 \mathrm{~J}$ ). No change in fatty acid oxidation, cholesterol synthesis, and LXR target gene expression were observed in liver of $E 2 f 1^{-1}$ mice compared with control mice (Supplemental Figure 7). Finally, we found that the hepatic expression of E2f1 in the HCD-fed mice was markedly augmented when compared with the chow-fed mice (Supplemental Figure 8), suggesting that E2f1 expression is diet dependent and plays a role in controlling homeostasis by regulating Pcsk 9 expression. Promoter luciferase experiments highlighted that SREBP2 and LRH1 participate in the transactivation of $E 2 F 1$ promoter, suggesting a possible molecular mechanism involving these transcription factors that promote E2f1 expression in response to an HCD (Supplemental Figure 9). In summary, these results supported the hypothesis that $E 2 f 1$ deletion in mice fed an HCD resulted in an increase in LDLR expression and cholesterol accumulation in the liver.

High dietary cholesterol promotes liver fibrosis in E2f1 $1^{-1-}$ mice. Morphological observations of the livers of E2f1 ${ }^{-/-}$mice suggested early signs of steatohepatitis development. Therefore, we performed trichrome and Sirius red staining on histological liver sections. Stronger staining was observed in the livers of the E2f1-1mice compared with the $E 2 f 1^{+/+}$mice, suggesting the presence of fibrosis in the liver of these mice fed the HCD (Figure 6A). Liver fibrosis is characterized by increased production of extracellular matrix, such as $\alpha$-collagen type I (Colla1). Furthermore, during the progression of liver fibrosis, hepatic stellate cells undergo transdifferentiation to a myofibroblast-like phenotype that expresses a high level of $\alpha$-smooth muscle actin ( $\alpha$-sma) (15). Accordingly, the mRNA levels of both fibrosis biomarker genes (Collal and $\alpha$ sma [Acta2]) and some hepatic stellate cell activation genes (Mmp2, Timp2) were also increased in the liver of $E 2 \mathrm{fl}^{-1-}$ mice (Figure 6B and Supplemental Figure 10). We checked inflammation gene markers, such as Il6 and Tnfa, and did not find any significant differences that could reveal a modification of inflammation between the $E 2 \mathrm{fl}^{+/+}$and $E 2 \mathrm{fl}^{-/-}$mice (Figure $6 \mathrm{~B}$ ). We also observed that the total BA levels in fecal material were decreased in $E 2 \mathrm{fl}^{-^{--}}$mice fed the HCD compared with $E 2 \mathrm{fl}^{+/+}$mice, suggesting that $E 2 f 1$ deletion led to an increase in cholesterol uptake and, possibly, a reduction in cholesterol breakdown to BA as previously published (16) (Figure 6C). No differences in fecal neutral sterol levels were detected between $E 2 \mathrm{fl}^{+/+}$and E2f1 ${ }^{-1-}$ mice (Supplemental Figure 11).

To determine if the reduction of $P c s k 9$ expression in $E 2 f 1^{-1-}$ mice contributes to the observed liver phenotype, we decided to perform a rescue experiment by reexpressing PCSK 9 in the liver of E2f1-1- mice by adenoviral infection. E2f1 ${ }^{-/-}$mice were injected with Ad-null or Ad-PCSK9 at week 2 of the 5-week HCD. Remarkably, the reexpression of PCSK 9 reversed the expression of fibrosis biomarkers genes (Colla1 and $\alpha$-sma [Acta2]), as well as Cd36 upregulation (Figure 6E). Consistently, at the histological level, we observed 
$A$

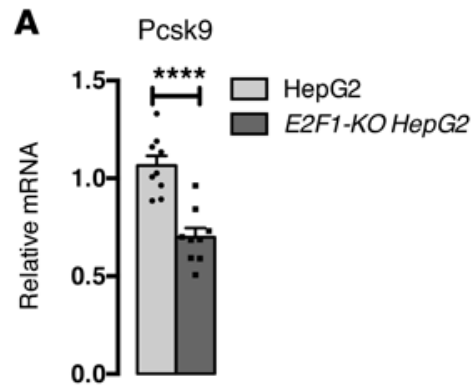

B

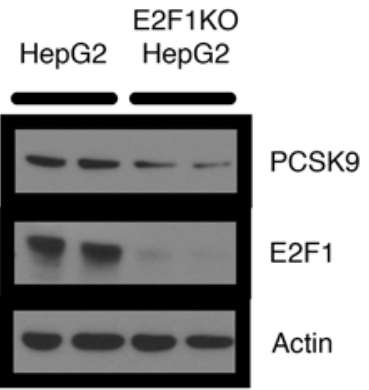

Westernblot

quantification $(n=3)$

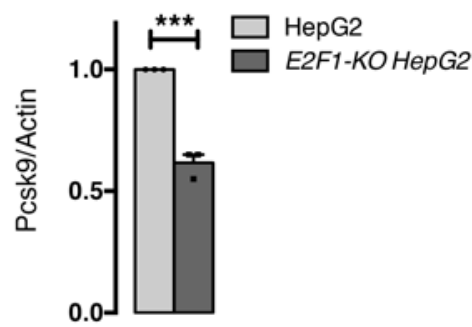

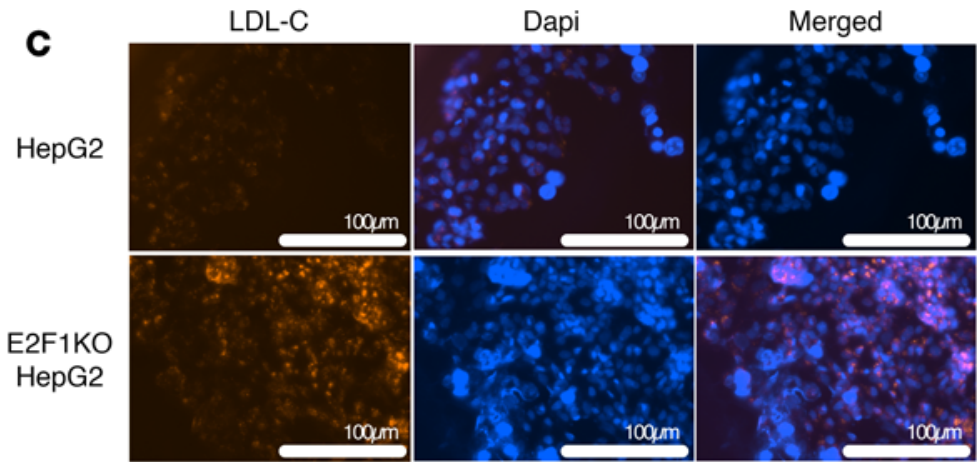

D

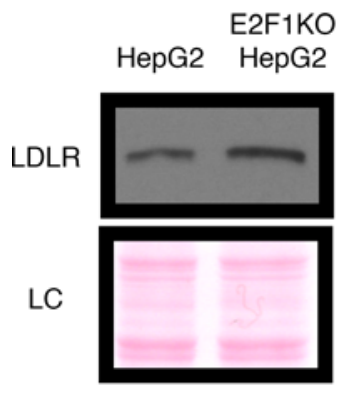

E

Cholesterol biosynthesis

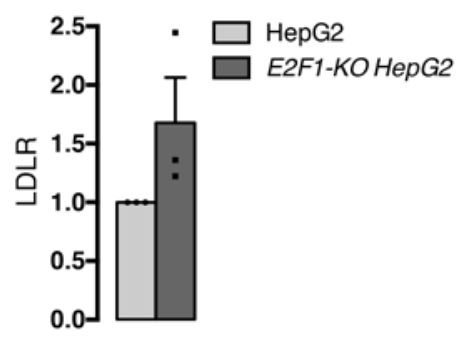

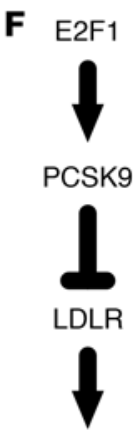

Reduce cholesterol uptake

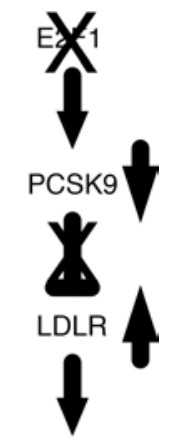

Increase cholesterol uptake

Figure 4. E2F1-KO HepG2 cells show increased LDL-cholesterol (LDL-C) uptake and decreased cholesterol biosynthesis. (A) Relative expression of $P C S K 9$ mRNA in HepG2 and E2F1-KO HepG2 cells. Three independent experiments in triplicate. (B) Representative images of PCSK9 Western blots from HepG2 and E2F1-KO HepC2 cells. Three independent experiments. Normalization of the quantified values is represented. (C) Representative images of LDL-C uptake in HepG2 and E2F1-KO HepG2 cells. Three independent experiments. Red fluorescence denotes LDL-C, and nuclei are stained in blue. Scale bars: $100 \mu \mathrm{m}$. (D) Representative images of LDLR Western blots from HepG2 and E2F1-KO HepG2 cell membranes. Three independent experiments. Ponceau staining is shown as loading control (LC). (E) Quantification of the rate of cholesterol biosynthesis in HepG2 and E2F1-KO HepC2 cells. Three independent experiments in triplicate. (F) Representative role of E2F1 in PCSK9-LDLR cholesterol uptake. All data are presented as the mean \pm SEM. Differences between HepG2 and E2F1-KO HepG2 were determined by 2-tailed unpaired $t$ test. ${ }^{* *} P<0.01,{ }^{* *} P<0.001$, ${ }^{* * *} P<0.0001$.

a reduction of Sirius red and Oil Red O staining in the liver, which is characteristic of a reduction of the progression of hepatic NAFLD (Figure 6D). These data suggested that E2F1 could protect mice from the early stages of steatohepatitis development by regulating optimal cholesterol uptake in a PCSK9-dependent manner (Figure 7).

Decreased levels of Pcsk9 in the E2f1-- genetic background resulted in liver injury in response to HCD. Since PCSK9 inhibitors have been recently approved by the FDA for the treatment of hypercholesterolemia, we next wanted to test if a reduction of PCSK9 in a WT background would also induce liver injury, similar to the lesions observed in E2f1 ${ }^{-/-}$livers. We therefore challenged Pcsk $9^{-/-}$mice with 6 weeks of HCD. As expected, Pcsk $9^{-/-}$mice showed reduced plasma cholesterol levels compared with $P c s k 9^{+/+}$mice (Figure 8A). At the end of the 6-week HCD treatment, we did not observe any morphological or fibrosis biomarkers increase in the livers of these mice (Figure 8, B and C). In agreement with a previous study that reported 
A

Plasma cholesterol

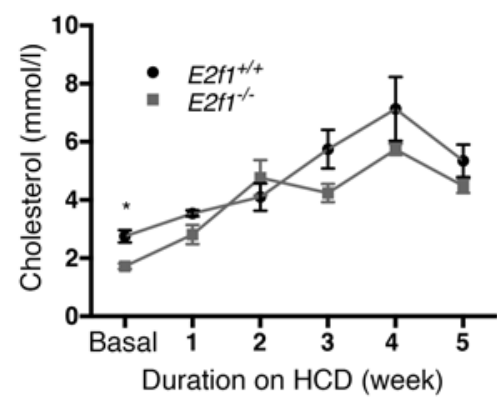

D $E 2 f 1^{+/ *}$

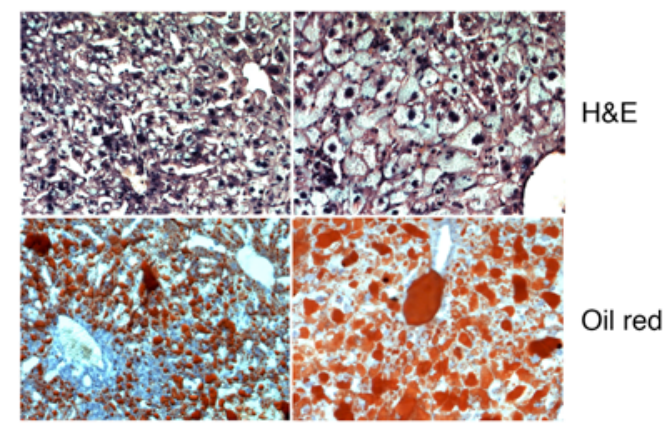

H

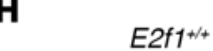

B

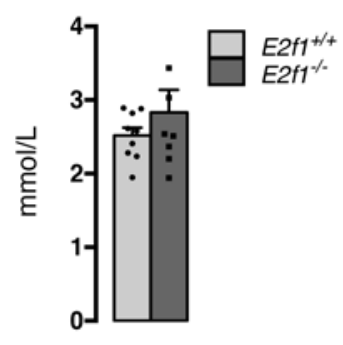

C Plasma LDL

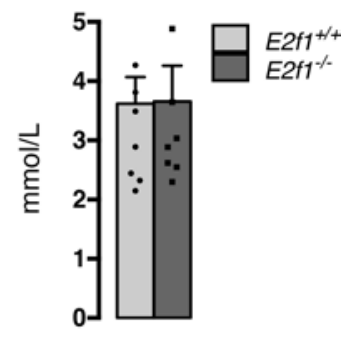

E

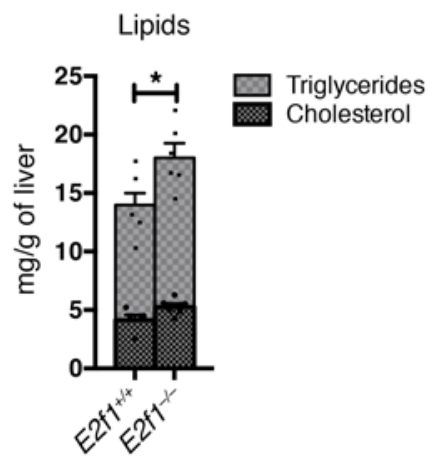

Free cholesterol

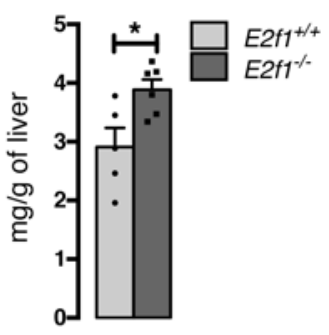

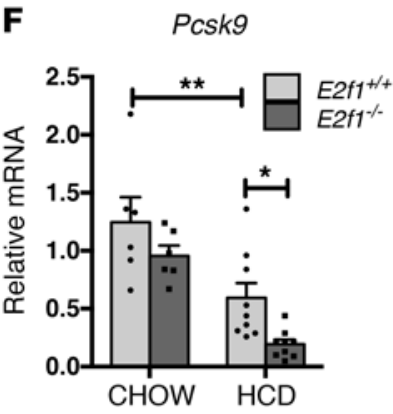

F

G

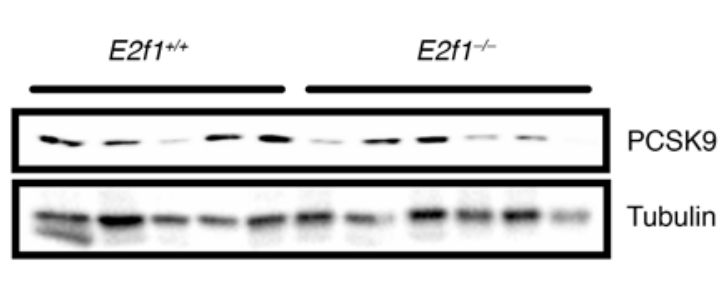

Quantification of

LDLR staining
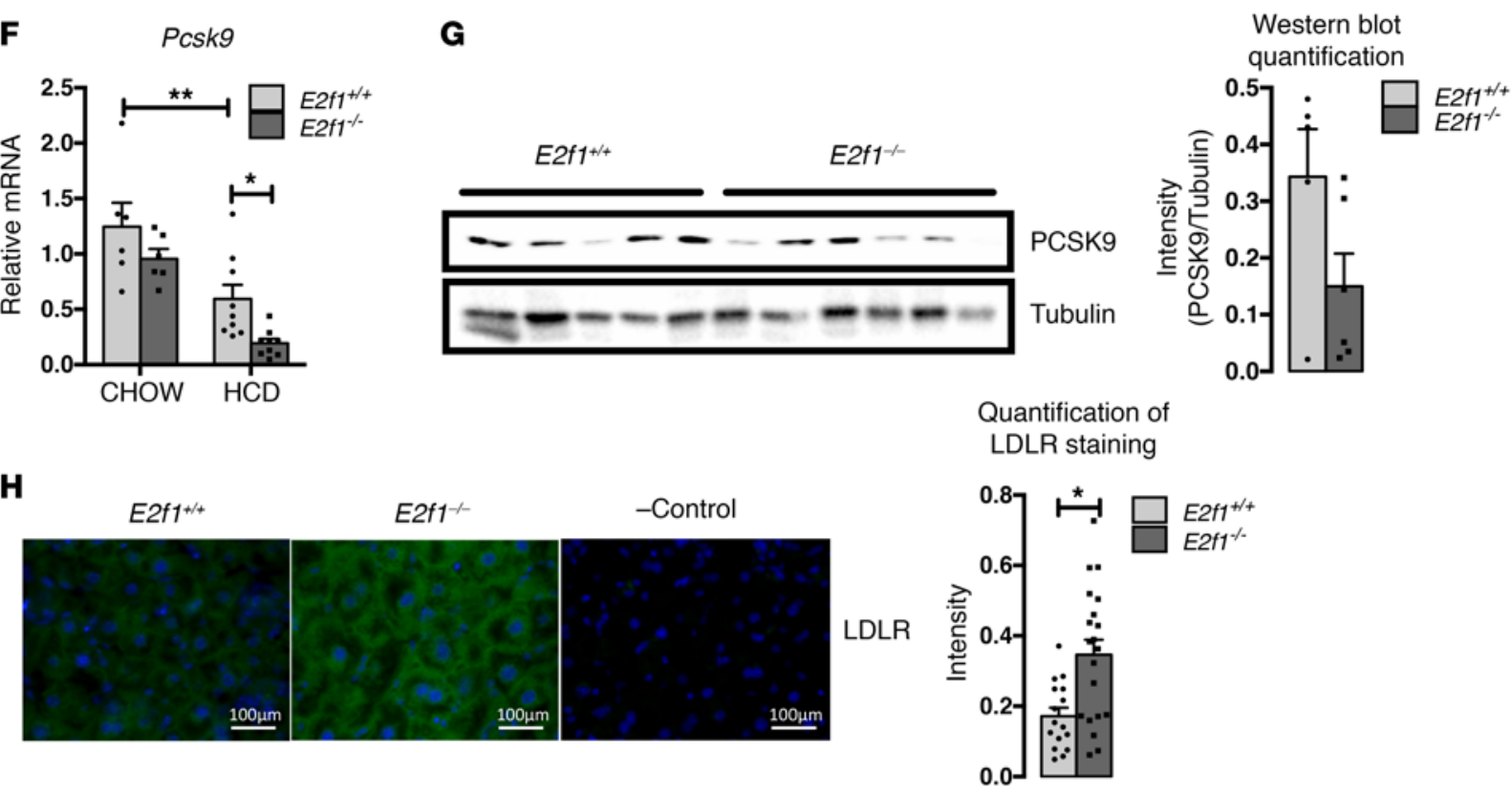

I

Western blot quantification

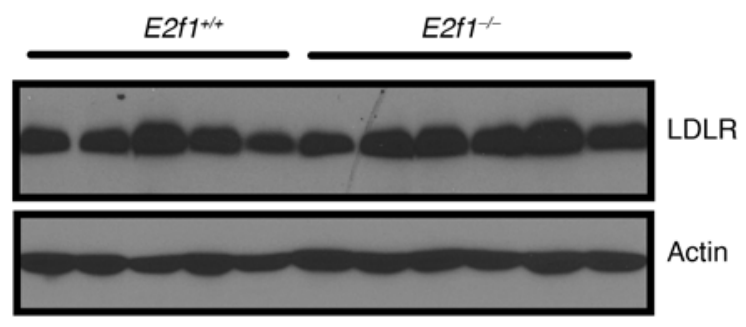

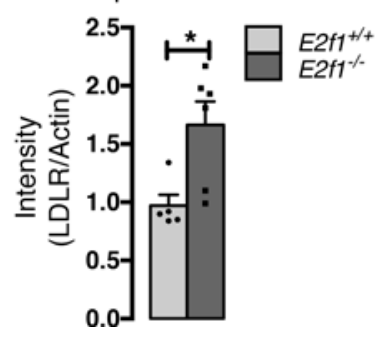

J $\quad C d 36$

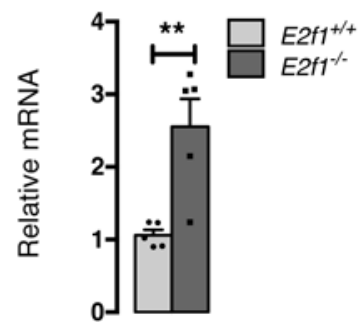


Figure 5. Cholesterol homeostasis and liver phenotype in E2F1-/- mice fed a high-cholesterol diet (HCD). (A) Plasma cholesterol profiles of $E 2 f 1^{+/+}$and E2 $\mathrm{f1}^{-/-}$mice before and after 5 weeks of being fed an HCD (1.25\% cholesterol). $n=3-6$ mice per group. (B and C) Plasma HDL and LDL-cholesterol levels after the 5 weeks of the HCD. $n=8-9$ mice per group. (D) H\&E and Oil Red 0 staining of $E 2 f 1^{+/+}$and $E 2 f 1^{-/-}$liver sections. Original magnification, $\times 100$. (E) Total lipid (triglycerides + cholesterol) and free cholesterol quantification in livers of $E 2 \mathrm{ft}^{+/+}$and $E 2 f 1^{-/-}$mice. $n=5-6$ mice per group. (F) Relative expression of Pcsk9 mRNA in livers from E2f1+/+ and E2f1-/- mice that were fed chow or HCD for 5 weeks. $n=6-9$ mice per group. Differences were determined by 2 -way ANOVA. ${ }^{*} P<0.05$, ${ }^{* *} P<0.01$. (G) Western blot of PCSK9 in the liver tissues of $E 2 \mathrm{f1}^{+/+}$and $E 2 \mathrm{f1}^{-/-}$mice that were fed an HCD for 5 weeks. Tubulin was used as a loading control. Normalization of the quantified values is represented. (H) Representative immunostaining of LDLR in the livers of $E 2 f 7^{+/+}$and $E 2 f 1^{-/-}$ mice that were fed an HCD. Scale bars: $100 \mu \mathrm{m}$. Quantification of LDLR staining is represented. Fluorescence intensity was normalized to the number of cells. (I) Western blot of LDLR in the liver tissues of E2 $\mathrm{f1}^{+/+}$and $E 2 \mathrm{f1^{-/- }}$ mice that were fed an HCD for 5 weeks. Actin was used as a loading control. Normalization of the quantified values is represented. (J) $C d 36$ gene expression in livers of $E 2 f 1^{+/+}$and $E 2 f 1^{-/-}$mice after 5 weeks of HCD. $n=5$ mice per group. All data are presented as the mean \pm SEM from a minimum of 5 mice per group. Differences between $E 2 \mathrm{fl}^{+/+}$and $E 2 f 1^{-/-}$were determined by 2 -tailed unpaired $t$ test, unless indicated otherwise. ${ }^{*} P<0.05,{ }^{* *} P<0.005$.

an increase in fecal BA excretion of $P c s k 9^{-1-}$ mice (17), we observed an increase in the expression of genes involved in BA synthesis (Cyp7b1, Cyp27aI), as well as the transporter Abcal and Abcg5 in the liver of Pcsk9--, compared with $P c s k 9^{+/+}$mice (Figure $8 \mathrm{D}$ ). This evidence suggests that, contrary to $E 2 f^{-/-}$mice, the modifications of BA metabolism in $P c s k 9^{-1-}$ mice protect against liver injuries, secondary to increased cholesterol uptake and accumulation.

\section{Discussion}

Dysregulation of lipid and lipoprotein metabolism is associated with NAFLD (18). NAFLD is the most common liver disease in the Western countries and affects approximately $30 \%$ of the US population. The pathology of NAFLD involves various stages, beginning with lipid accumulation in the liver (simple steatosis) and progressing to chronic inflammation that may lead to nonalcoholic steatohepatitis (NASH), hepatic fibrosis, cirrhosis, and hepatocellular carcinoma (19). Although the underlying mechanism that induces inflammation and fibrosis remains unclear, several studies have established that perturbed cholesterol homeostasis is central to the development of NASH $(20,21)$. Here, we show that the transcription factor E2F1, which is primarily known for its function in cell cycle regulation, also participates in cholesterol homeostasis. We identified a new transcriptional target of E2F1, Pcsk9, which is characterized by playing a role in the regulation of LDLR degradation and cholesterol uptake. Consequently, E2f1 ${ }^{-/-}$mice display reduced $P c s k 9$ expression in the liver, leading to a decrease in plasma total cholesterol levels and an increase in the cholesterol content of the liver and colon. These results initially led us to consider that E2fI deletion might prevent cardiovascular disease (CVD). Surprisingly, further analysis highlighted that hypercholesterolemia was not prevented by the lack of E2F1 in response to an HCD (Figure 5A). Instead, likely due to excessive cellular cholesterol accumulation, $E 2 \mathrm{fl}^{-/-}$mice presented early signs of liver fibrosis, which is reversed by PCSK9 reexpression (Figure 6). Even if PCSK9 rescues the effects on cholesterol-induced liver injury in $E 2 \mathrm{ft}^{-/}$mice, we cannot exclude that other factors participated in the phenotype observed by E2F1 systemic loss. Indeed, our results suggested that liver fatty acid uptake, via CD36, could participate in hepatic steatosis (Figure 5J), as well as reduced cholesterol excretion in the form of BA (Figure 6C and Supplemental Figure 5).

E2F1 has recently been implicated in liver fibrosis (16) in a study in which Zhang and coworkers detected increased E2F1 expression in human fibrotic/cirrhotic livers and in a mouse model of hepatobiliary injury. We observed similar E2f1 induction in the livers of mice fed an HCD (Supplemental Figure 8). Nevertheless, in contrast to our observations, the authors reported that $E 2 f 1^{-/-}$mice were protected from liver fibrosis induced by 3,5-diethoxycarbonyl-1,4-dihydrocollidine (DDC) supplementation. Fibrosis initiation in this specific model (DCC) is dependent on BA accumulation and toxicity and not on lipid accumulation, as observed when mice are fed an HCD diet, which may explain the differences observed between these 2 models.

We showed that E2F1 regulates Pcsk 9 mRNA expression, which is a promising therapeutic target for lowering plasma LDL-C levels. Indeed, recent clinical trials revealed that treatment with a PCSK9 inhibitor was effective in clearing as much as $70 \%$ of plasma LDL-C, with a good safety profile (22). The transcriptional regulation of $P c s k 9$ relies largely on the nutritional status and cholesterol cellular content. Other transcription factors, including SREBP2, SREBP1c, and HNF1 $\alpha$, control PCSK9 expression $(13,23,24)$. Interestingly, we previously showed that E2F1 is activated by insulin and controls Srebp-1c gene expression in the liver (5), suggesting that cross-talk exists between these pathways. 
A

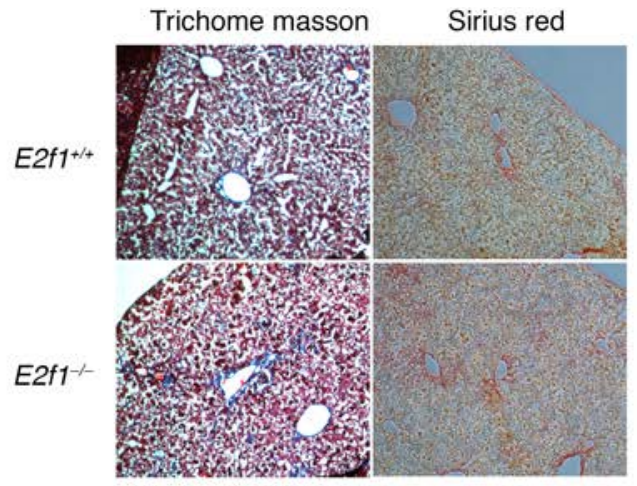

C

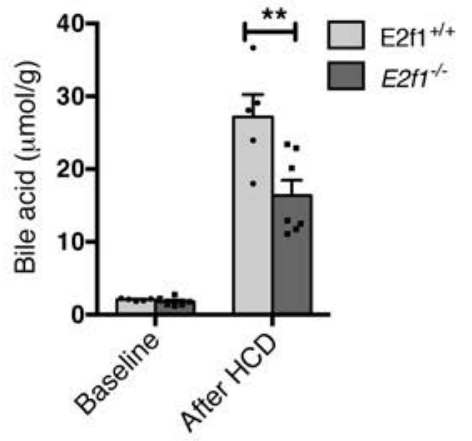

B

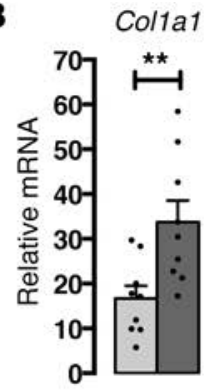

116
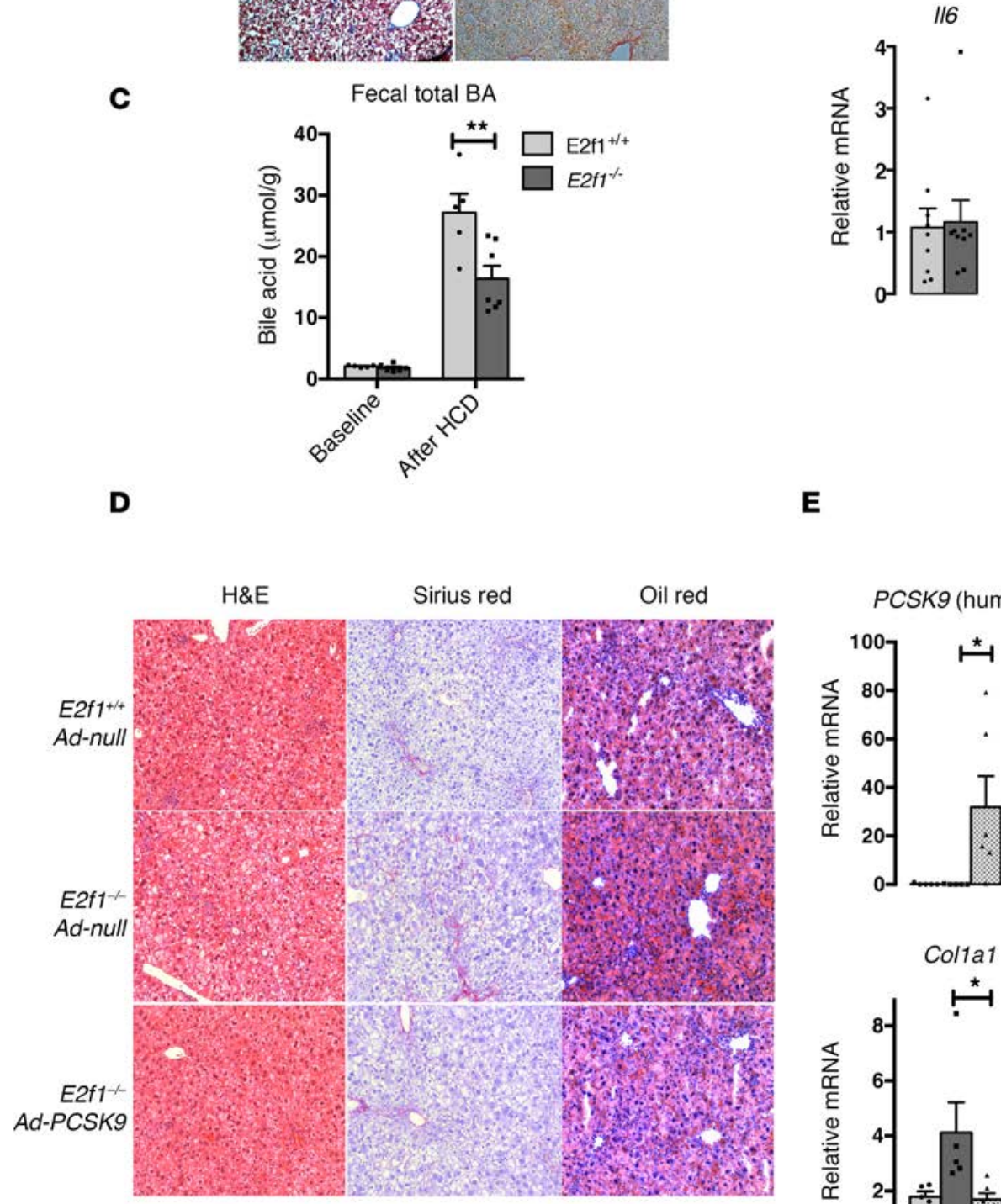
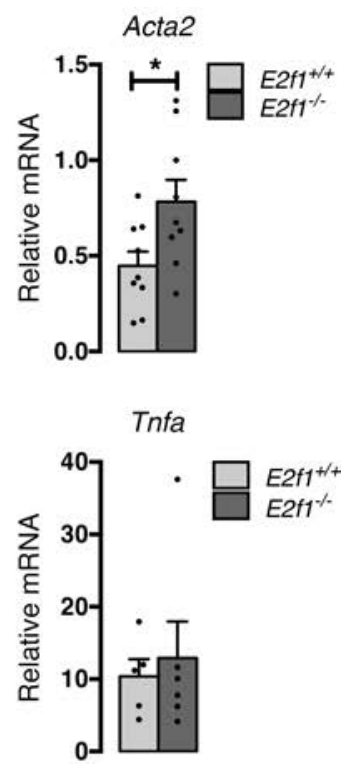

E

Oil red
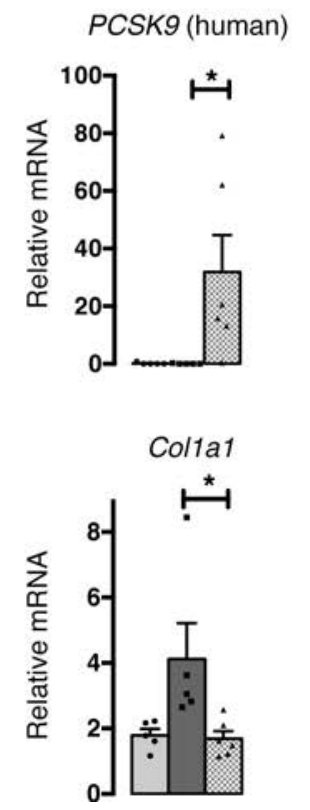

D

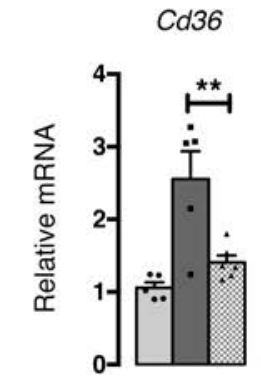

Figure 6. E2f1/-- mice exhibit more pronounced liver fibrosis after 5 weeks on the high-cholesterol diet (HCD). Expression of PCSK9 protect liver of E2f1 ${ }^{-/-}$ mice from liver injuries. (A) Representative images of trichrome staining and Sirius red staining of livers from the $E 2 \mathrm{f1}^{+/+}$and $E 2 \mathrm{f1}^{-/-}$mice that were fed an HCD for 5 weeks. Blue denotes collagen staining. Scale bar: $100 \mu \mathrm{m}$. (B) Relative mRNA expression of fibrosis-inflammation-related genes in the liver

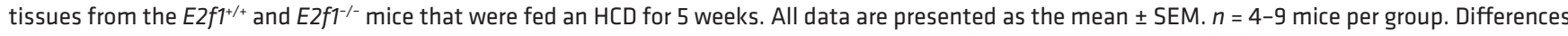
between $E 2 \mathrm{fl}^{+/+}$and $E 2 \mathrm{f1}^{-/-}$were determined by 2-tailed unpaired $t$ test. ${ }^{*} P<0.05$, ${ }^{* *} P<0.01$. (C) Fecal total bile acid (BA) quantification of $E 2 f 1^{+/+}$and $E 2 f 1^{-/-}$mice before and after 5 weeks of HCD (5-7 samples per group). Differences between $E 2 f 1^{+/+}$and E2f1-/- were determined by 2-way ANOVA. ${ }^{* *} P<$

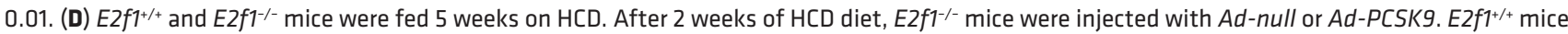
injected with $A d$-null are used as control. Representative images of H\&E staining, Sirius red staining, and Oil Red $O$ staining of liver sections. Original magnification, $\times 100$. (E) Relative mRNA expression of $P C S K 9$, fibrosis-related genes, and $C d 36$ in the liver tissues from the $E 2 \mathrm{f1}^{1^{/+}}$and $E 2 \mathrm{f1}^{-/-}$mice fed on HCD and injected with Ad-null or Ad-PCSK9 as indicated. All data are presented as the mean \pm SEM. $n=4-6$ mice per group. Differences between $E 2 f 1^{-1-}$ Ad-null and E2f1-1- Ad-PCSK9 were determined by one-way ANOVA. ${ }^{*} P<0.05,{ }^{* *} P<0.01$. 


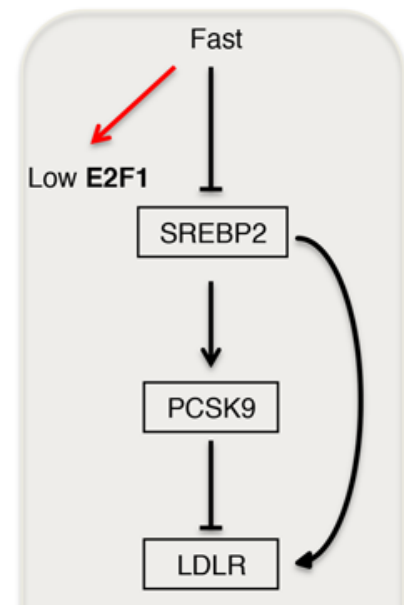

I

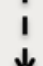

- Low PCSK9

- High LDLR

- Synthesis inactivated

- LDL-C uptake High

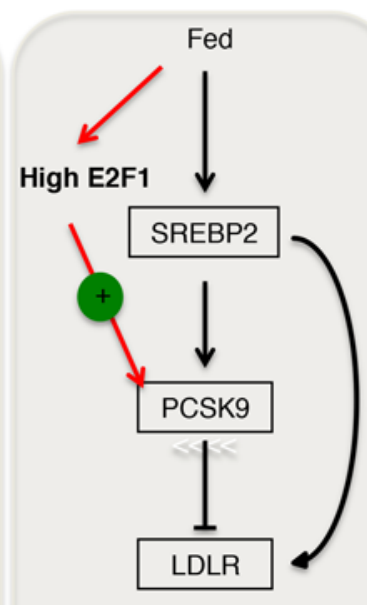

I

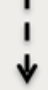

- High PCSK9

- Low LDLR

- Synthesis activated

- LDL-C uptake low

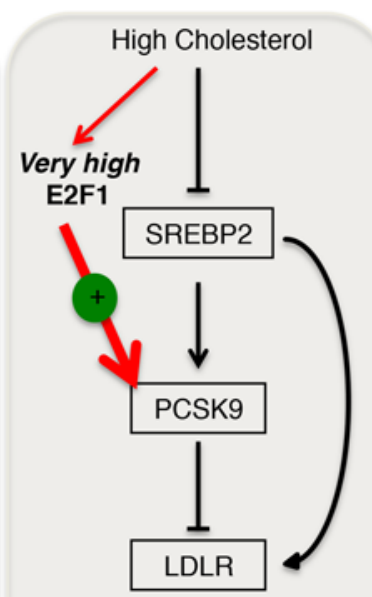

I

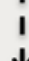

- $\quad$ Moderate PCSK9

- Moderate LDL-C uptake
- Moderate LDLR

- Synthesis inactivated

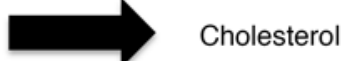

- Very Low PCSK9

- Very High LDLR

- Synthesis blocked

- LDL-C uptake very high

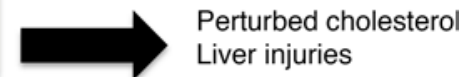

- $\quad$ SDLnthesis blocked
- High CD36

- Not efficient BA excretion

Figure 7. Proposed model of E2F1 regulation in response to diets. E2F1 expression is diet dependent. E2F1 and SREBP2 work in a synergistic manner to regulate LDLR availability to PCSK9 and consequently LDL-cholesterol uptake. Fast, fasted state; fed, fed state, high cholesterol, cholesterol-overloaded diet. Effects of E2F1 invalidation on cholesterol metabolism under physiological and pathological conditions is shown.

In the present work, we have illustrated that hepatic $P c s k 9$ mRNA expression is reduced in response to an $\mathrm{HCD}$ compared with a normal chow diet (Figure $5 \mathrm{~F}$ ), suggesting that under these conditions, PCSK9 inhibition is required to increase LDLR activity and, thus, cholesterol uptake. Interestingly, when mice were challenged with an HCD, Pcsk 9 expression was further decreased in $E 2 \mathrm{fl}^{-{ }^{--}}$mice. Strikingly, we found that hepatic E2f1 expression was increased in response to the HCD compared with the chow diet. Based on these results, we can conclude that induction of E2F1 in mice fed an HCD is required to repress LDLR-mediated cholesterol uptake through PCSK9 activation. Further supporting this conclusion, when E2f1 was deleted and mice were fed the HCD, hepatic Pcsk9 expression was detected at very low levels, and reexpression of PCSK 9 can protect the liver in these conditions. Hence, we can speculate that E2F1-mediated regulation of Pcsk 9 expression is a compensatory mechanism to avoid excessive cholesterol accumulation in cells. Indeed, excess intracellular cholesterol levels are toxic to cells (25). However, the obvious effect of decreased Pcsk9 expression is a reduction in plasma LDL-C levels. PCSK9 monoclonal antibodies are approved today for the treatment of CVDs (26). Most crucially, our data highlight the observation that, although inhibiting PCSK9 is beneficial for reducing circulating LDLC levels, efficient uptake by the LDL system must be accompanied by a competent sterol excretion system, like BA metabolism, to avoid excessive cholesterol accumulation in the liver. E2f1 deficiency is critical for cholesterol uptake and BA synthesis and excretion to conserve liver integrity under pathological conditions (16). In contrast, Pcsk 9 invalidation in the $E 2 f^{1^{++}}$genetic background promotes both cholesterol uptake and BA excretion (Figure 8). As a consequence, PCSK9 genetic inhibition did not lead to increased hepatic steatosis under high-cholesterol overload. Indeed, PCSK9 invalidation alone is not able to promote cholesterol-induced liver fibrosis, which could be explained 
A

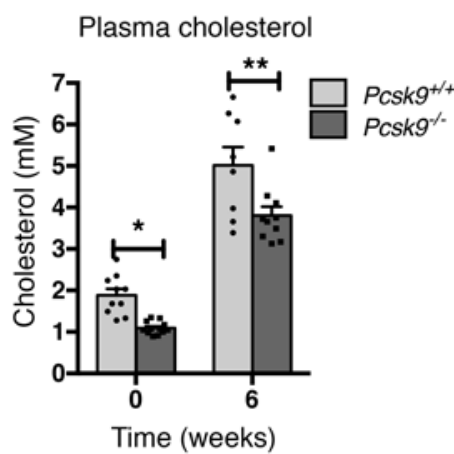

B
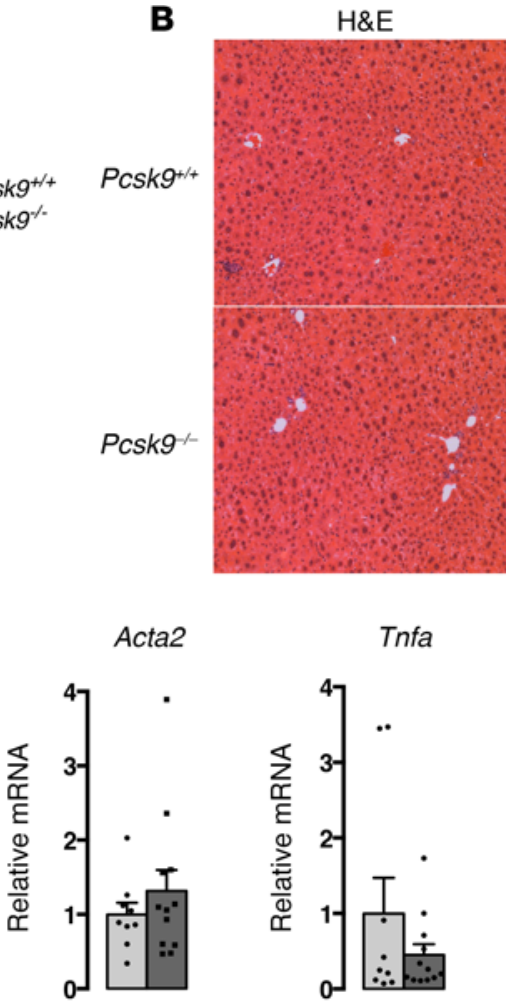

Cyp27a1

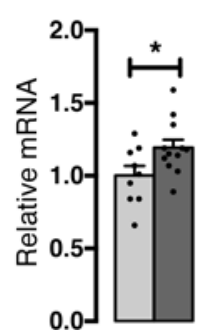

Tnfa

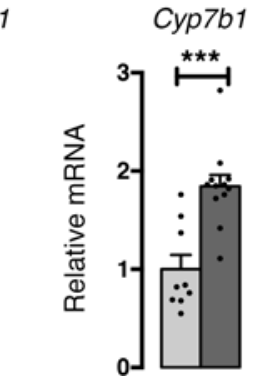

Col1a1

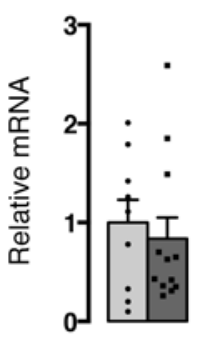

D Cyp7a1
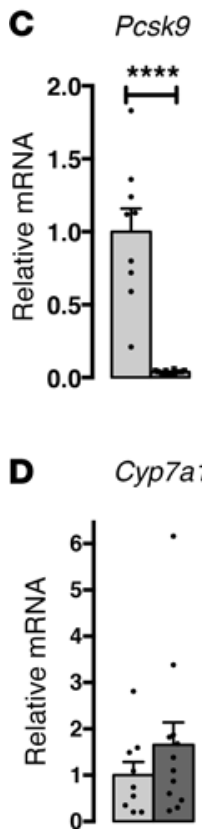

Sirius red

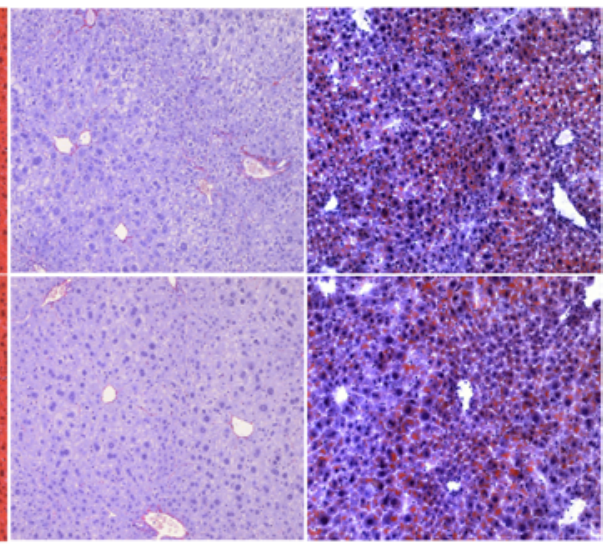

116

Figure 8. Pcsk9-/- mice does not present liver injury after 6 weeks on the high-cholesterol diet (HCD). (A) Plasma cholesterol profiles of $P c s k 9^{+/+}$and $P c s \mathrm{~kg}^{-/-}$mice before and after 6 weeks of HCD. Differences between $P c s \mathrm{~kg}^{+/+}$and $P c s k 9^{-/-}$were determined by 2 -way ANOVA. ${ }^{*} P<0.05,{ }^{* *} P<0.005$. (B) $\mathrm{H} \& \mathrm{E}$, Sirius red, and Oil Red $\mathrm{O}$ staining of $P c s \mathrm{~kg}^{+/+}$and $P c s \mathrm{~kg}^{-/-}$liver sections. Original magnification 100x. (C and D) Relative expression of PCSK9, fibrosis-inflammation related genes, and bile acid (BA) metabolism-related genes in the liver of $P c s k 9^{+/+}$and $P c s k 9^{-/-}$mice on $\mathrm{HCD}$. $n=9-12 \mathrm{mice}$ per group. Differences between $P \mathrm{csk} \mathrm{g}^{+/+}$and $P \mathrm{csk} \mathrm{kg}^{-/-}$were determined by 2-tailed unpaired $t$ test. ${ }^{*} P<0.05,{ }^{* *} P<0.005,{ }^{* * *} P<0.0005,{ }^{* * * *} P<0.0001$.

by improvement of BA metabolism (Figure 8) (17). Further studies are needed to explore the mechanisms involved. More importantly, the comparison of our 2 mice models $\left(E 2 f 1^{-/-}\right.$and Pcsk $\left.{ }^{-1-}\right)$ on HCD revealed the importance to conserve a liver metabolic flexibility to avoid liver cholesterol accumulation and preserve liver integrity.

In conclusion, our study supports the hypothesis that E2F1-induced Pcsk9 expression is important to moderately control the amount of LDLR and support cellular cholesterol homeostasis (Figure 7). In our model, we established that E2F1 regulates the availability of the LDLR through PCSK9 in response to dietary status. In addition to SREBP2 activation, high levels of E2F1 expression during feeding conditions induce PCSK9 expression, which in turn reduces available LDLR levels. This mechanism prevents cholesterol that is simultaneously derived from LDLR-mediated uptake and synthesis from overloading cells, maintaining the whole-body cholesterol balance. However, during high-cholesterol dietary intake, SREBP2 activity is inhibited to limit cholesterol loading from the synthesis and uptake pathways. In this case, E2F1 acts as an alternative regulator to control optimal LDLR-mediated sterol clearance through PCSK9. 


\section{Methods}

Animal experiments. E2f1 ${ }^{+/+}$and $E 2 f 1^{-/-}$mice $(B 6 ; 129 S 4-E 2 f 1 \mathrm{tm} 1 \mathrm{Meg} / \mathrm{J})$ were purchased from The Jackson Laboratory. $P c s k 9^{+/+}$and $P c s k 9^{-/-}$were obtained from Bertrand Cariou and Cédric Le May (27). Mice were fed normal chow or an HCD (catalog TD.88051, Harlan) and housed under a 12-hour light/dark cycle. For rescue experiment, after 2 weeks of $\mathrm{HCD}, E 2 f 1^{+/+}$and $E 2 f 1^{-/-}$mice were injected with Ad-null and Ad-PCSK9, expressing human PCSK9 (28). Then the mice were fed 3 more weeks on HCD. Adenoviruses were provided by Bertrand Cariou and Cédric Le May. All mice in the fed and overnight-fasting groups were euthanized at $10 \mathrm{am}$. Organs and blood samples were collected for all experiments.

Cell culture and transfection. The HepG2 cell line was purchased from the American Type Culture Collection (ATCC) and cultured in 10\% FBS DMEM. E2F1 was knocked out using CRISPR/Cas9 technology. LentiCRISPR v2 was a gift from Feng Zhang (Cambridge University, Cambridge, England) (Addgene plasmid 52961); a description of the plasmid can be found in Sanjana, Shalem et al. 2014 (29). Both the pMD2.G and psPAX2 plasmids were gifts from Didier Trono (University of Lausanne) (Addgene plasmid 12259 and 12260, respectively). The target sequences for the E2F1 guide were 5'CACCGTCTGACCACCAAGCGCTTCC-3' and 5'-AACGGAAGCGCTTGGTGGTCAGAC-3'. The oligos were synthesized and cloned into the digested LentiCrispR vector in accordance with the protocol described by Shalem et al. (29). Lentiviral production was based on the standard protocol established by Salmon and Trono (30). For lentivirus infection, HepG2 cells were infected with $2 \mathrm{ml}$ of the obtained virus for 72 hours. Infected HepG2 cells were treated with $5 \mu \mathrm{g} / \mathrm{ml}$ puromycin for 5 consecutive days, with fresh medium (DMEM/Glutamax plus 10\% FBS) added every 2-3 days during the treatment period to select for the positive, infected cells. Western blotting was performed to ensure that the E2F1 protein (catalog Sc-193, Santa Cruz Biotechnology Inc.) was not expressed. For the experiments, we treated the cells with either 10\% delipidated FBS (DL) (catalog S5394, Sigma-Aldrich) or 10\% FBS. The HepG2 cell line was transfected using X-tremeGene (catalog 06366236001, Roche Diagnostics). Human PCSK9 promoter reporter plasmid, control pLightSwitch_Prom plasmid, and human E2F1 promoter reporter plasmid were purchased from SwitchGear (catalog S712669, catalog S790005, and catalog S719961, respectively). Human PCSK9 promoter (920 pb, -647 to +273 of the transcription start site [TSS]) was cloned in pLightSwitch_Prom plasmid.

We isolated mouse primary hepatocytes in accordance with the protocol described in Benhamed et al. (31). For the experiments, we treated the cells with DMSO (control) or $0.065 \mathrm{mM}$ water-soluble cholesterol (catalog C4951, Sigma-Aldrich) overnight.

$E 2 F 1^{+/+}$and $E 2 F 1^{-/-}$MEFs were derived from embryos at 13.5 days after vaginal plugs as previously described (32).

Histology. Liver tissues were fixed in $4 \%$ neutral buffered formalin and embedded in OCT freezing medium. The tissues were cut and stained with H\&E for morphological analysis, Oil Red $\mathrm{O}$ to detect neutral lipids or trichrome, and Sirius red to detect fibrosis. The tissues were sectioned and stained by the Mouse Pathology Facility at UNIL, Switzerland, and Catherine Moret at the Center for integrative Genomics (CIG), University of Lausanne (UNIL), Switzerland.

Fecal analysis. Feces of singly housed mice or pooled feces from $E 2 f 1^{+/+}$and $E 2 f 1^{-/-}$mice were collected after 24 hours and dried at $70^{\circ} \mathrm{C}$. Fecal samples were used to quantify BA and neutral sterol levels using gas-liquid chromatography-mass spectrometry, as previously described (33) or as otherwise indicated using a commercial kit (catalog 80470, Crystal Chem; catalog 1 1300, Diasys). All gas-liquid chromatography-mass spectrometry analyses were performed by Albert K Groen at the University of Groningen, The Netherlands.

$q P C R$ analysis. Total mRNA was extracted from $20-30 \mathrm{mg}$ of liver or cultured cells using an RNeasy kit (catalog 74106, Qiagen) according to the manufacturer's protocol. RNA (1 $\mu \mathrm{g})$ was subsequently reverse-transcribed and quantified via qPCR using an ABI 7900HT instrument. All presented data were based on the standard curve method and normalized to mouse RS9 levels.

ChIP experiment. ChIP was performed as previously described (5). Briefly, cells were fixed with a $1 \%$ formaldehyde solution and lysed with a cell lysis solution. The isolated nuclear extract was sheared through 20 cycles of sonication (30 seconds on, 30 seconds off per cycle). A DNA gel was run to verify that isolated chromatin was sheared into small fragments spanning approximately 300-800 bp. Magnetic beads were precleared with BSA and salmon sperm, followed by incubation with the E2F1 antibody (catalog sc-193, Santa Cruz Biotechnology Inc.) for 1 hour at $4^{\circ} \mathrm{C}$ with rotation. The isolated chromatin was immunoprecipitated with the prepared magnetic beads overnight at $4^{\circ} \mathrm{C}$. The immunoprecipitated chromatin 
was washed and, together with the input samples, reverse cross-linked at $65^{\circ} \mathrm{C}$ overnight. The DNA was subsequently purified with a MinElute column (catalog 28206, Qiagen). Finally, E2F1 DNA binding was quantified via qPCR.

High-throughput ChIP-seq. The ChIP-seq experiment was performed as previously described (5). The sequencing data are available in the NCBI Gene Expression Omnibus (GEO) database (GEO GSE74006) (5).

Lipid analysis. Lipids were extracted with acetone as previously described (34) or by using the Folch method (35). Briefly, 30-50 mg of liver tissue were ground in $1 \mathrm{ml}$ of PBS, followed by the addition of $5 \mathrm{ml}$ of a chloroform/methanol (2:1) solution. The sample was vortexed vigorously and centrifuged at 10,000 $g$. The lower chloroform phase containing the lipids was transferred to a new glass tube, and the sample was dried using nitrogen gas. Cholesterol and triglyceride concentrations were quantified with commercial kits (catalogs 113009910026 and DIA0060, Diasys). Fast protein liquid chromatography (FPLC) was performed as previously described (27).

LDL-C uptake assay. LDL-C uptake was determined according to the manufacturer's protocol (catalog ab133127, Abcam). Briefly, 4,000 E2f1 ${ }^{+/+}$and $E 2 f 1^{-/-}$cells/well were plated in 96-well plates and cultured in medium supplemented with $10 \%$ FBS or $10 \%$ delipidated FBS for 24 hours. After treatment, $75 \mu 1$ of diluted LDL-DyLight 550 was added to each well, followed by incubation for 4 hours at $37^{\circ} \mathrm{C}$ in a $5 \% \mathrm{CO}_{2}$ incubator and subsequent cell fixation. All images were captured using Biotek Cytation 3 at 200× magnification. The images were quantified using Fiji software.

Cholesterol biosynthesis assay. Approximately $5 \times 10^{5}$ cells were plated in 6-well plates and treated overnight. After treatment, the cells were washed twice with PBS. Fresh serum-free medium supplemented with $2 \mu \mathrm{Ci}$ of tritium-labeled acetic acid and $2.5 \mathrm{mM}$ cold-labeled sodium acetate (catalog S5636, Sigma-A1drich) was added to all wells, followed by incubation for 4 hours at $37^{\circ} \mathrm{C}$ in a $5 \% \mathrm{CO}_{2}$ incubator. After a 4-hour incubation, the cells were scraped in $500 \mu \mathrm{l}$ of ice-cold $2 \% \mathrm{NaCl}$, and $0.045 \mu \mathrm{Ci}$ of $\mathrm{C} 14$ radiolabeled cholesterol was added to the cell lysate as an internal control. Lipids were isolated from $400 \mu 1$ of the cell lysate using Folch's method (chloroform/methanol, 2:1), and the remaining $100 \mu 1$ of the cell lysate was used to quantify protein concentrations. The isolated lipids were dried under nitrogen gas and resuspended in $150 \mu 1$ of Folch reagent. The sample ( $50 \mu 1)$ was loaded onto a TLC silica plate, and the plate was stained with iodine vapor following the separation of lipids. The cholesterol band was identified based on comigration with a cholesterol standard. The cholesterol bands were excised and placed into glass scintillation vials to measure tritium and C14 radioactivity using a liquid scintillation counter. The results were normalized to the amount of protein and C14 counts (internal control).

Immunoblotting. Cellular membrane extracts were prepared using the Mem-PER Plus memebrane Protein Extraction Kit (catalog 89842, Thermo Fisher Scientific), and total protein extracts were performed as previously described (31). Protein samples were separated via SDS-PAGE and transferred to a nitrocellulose membrane. The membrane was blocked in 5\% milk diluted in tris-buffered saline-Tween 20 and incubated with a primary antibody overnight at $4^{\circ} \mathrm{C}$. The blot was subsequently washed and incubated with the corresponding secondary antibody for 1 hour at room temperature (RT) with shaking. The immunoblot was revealed using ECL and imaged using ChemiDoc XRS software or film. The following antibodies were used: E2F1 (catalog sc-193, Santa Cruz Biotechnology Inc.), LDLR (catalog ab30532, Abcam), PCSK9 (catalog ab31762, Abcam), $\beta$-actin (catalog A2066, Sigma-Aldrich), and $\alpha$-tubulin (catalog T6199, Sigma-Aldrich).

LDLR immunostaining. The mouse liver was cryosectioned at a thickness of $10 \mu \mathrm{m}$. Tissue sections were fixed in $4 \%$ formalin and blocked in 1\% BSA/TBST for 1 hour. The LDLR primary antibody (catalog ab30532, Abcam) was diluted in blocking solution, followed by incubation overnight at $4^{\circ} \mathrm{C}$. After incubation, the tissue sections were washed 3 times in PBS and incubated with an Alexa Fluor 488-conjugated anti-rabbit IgG antibody (catalog A21206, Invitrogen) for 1 hour at RT. The tissue sections were subsequently washed 3 times in PBS, incubated with DAPI for 3 minutes, and then washed again. The samples were mounted using mounting medium and analyzed under a fluorescent microscope.

Filipin staining. Cultured cells or mouse liver cryosections were fixed in $4 \%$ formalin for 10 minutes at RT. The samples were washed 3 times with PBS and incubated with $1.5 \mathrm{mg}$ glycine/ $\mathrm{ml}$ PBS for 10 minutes at RT to quench the formalin. The samples were subsequently stained with the filipin working solution (0.05 $\mathrm{mg} / \mathrm{ml}$ in PBS) for 2 hours at RT. Then, the samples were washed with PBS and viewed under a fluorescence microscope. 
Statistics. All data are expressed as the mean \pm SEM. Statistical significance was assessed with unpaired $t$ test or 2-way ANOVA, as appropriate, using Prism 6 software (GraphPad Software). Differences were considered statistically significant at $P<0.05$.

Study approval. All animal experiments were performed in accordance with Swiss animal welfare laws and approved by the Canton of Vaud SCAV (authorization VD2627 and VD3046).

\section{Author contributions}

QL designed the project, performed the experiments, assembled the data, analyzed and interpreted the results, and wrote and approved the manuscript. CLM and BC performed the FPLC analysis and provided Ad-pcsk9 and $\mathrm{PCSK}^{-/-}$mice. AG performed experiments in MEFs, primary culture of hepatocytes and mice. LZ performed adenoviral injection. LF and PDD designed the project and wrote and approved the manuscript. All authors read and provided comments on the manuscript

\section{Acknowledgments}

We acknowledge the members of the Fajas laboratory for their support and discussions. We thank Gilles Willemin from the Mouse Metabolic Facility of the University of Lausanne for the lipid plasma analysis; Albert K Groen (Bert) from the University of Groningen for measuring the fecal neutral sterol and BA levels; Stehle Jean and Horlbeck Janine from the Mouse Pathology Facility of the University of Lausanne and Catherine Moret from the Center for Integrative Genomics of the University of Lausanne for tissues histology sectioning and staining; Anne-Catherine Thomas for genotyping; and Fanny Thévenaz and colleagues from the Animal Facility of the University of Lausanne DNF-DP for taking care of the mice. BC and CLM received funding from the Fondation Leducq (grant 13CVD03). This work was supported by the Swiss National Fund (SNF) and the Fondation Suisse de Cardiologie.

Address correspondence to: Lluis Fajas or Pierre-Damien Denechaud, University of Lausanne, Center for Integrative Genomics, Quartier UNIL-Sorge, Bat. Genopode, CH-1015 Lausanne, Switzerland. Phone: 41.21.692.4111; E-mail: Lluis.Fajas@unil.ch (L. Fajas). Phone: 41.21.692.4139; E-mail: pierre-damien.denechaud@unil.ch (P.D. Denechaud).

1. Biswas AK, Johnson DG. Transcriptional and nontranscriptional functions of E2F1 in response to DNA damage. Cancer Res. 2012;72(1):13-17.

2. Dyson N. The regulation of E2F by pRB-family proteins. Genes Dev. 1998;12(15):2245-2262.

3. Frolov MV, Dyson NJ. Molecular mechanisms of E2F-dependent activation and pRB-mediated repression. J Cell Sci. 2004;117(Pt 11):2173-2181

4. Blanchet E, et al. E2F transcription factor-1 regulates oxidative metabolism. Nat Cell Biol. 2011;13(9):1146-1152.

5. Denechaud PD, et al. E2F1 mediates sustained lipogenesis and contributes to hepatic steatosis. J Clin Invest. 2016;126(1):137-150.

6. Fajas L, Landsberg RL, Huss-Garcia Y, Sardet C, Lees JA, Auwerx J. E2Fs regulate adipocyte differentiation. Dev Cell. 2002;3(1):39-49.

7. Hsieh MC, Das D, Sambandam N, Zhang MQ, Nahlé Z. Regulation of the PDK4 isozyme by the Rb-E2F1 complex. J Biol Chem. 2008;283(41):27410-27417.

8. Accad M, Farese RV. Cholesterol homeostasis: a role for oxysterols. Curr Biol. 1998;8(17):R601-R604.

9. Brown MS, Goldstein JL. A receptor-mediated pathway for cholesterol homeostasis. Science. 1986;232(4746):34-47.

10. Ishibashi S, Brown MS, Goldstein JL, Gerard RD, Hammer RE, Herz J. Hypercholesterolemia in low density lipoprotein receptor knockout mice and its reversal by adenovirus-mediated gene delivery. J Clin Invest. 1993;92(2):883-893.

11. Borghi C, Cimminiello C. [The new lipid-lowering drugs: focus on monoclonal antibodies]. G Ital Cardiol (Rome). 2016;17(4 Suppl 1):14S-121.

12. Lipari MT, et al. Furin-cleaved proprotein convertase subtilisin/kexin type 9 (PCSK9) is active and modulates low density lipoprotein receptor and serum cholesterol levels. J Biol Chem. 2012;287(52):43482-43491.

13. Costet $\mathrm{P}$, et al. Hepatic PCSK9 expression is regulated by nutritional status via insulin and sterol regulatory element-binding protein 1c. J Biol Chem. 2006;281(10):6211-6218.

14. Yu L, et al. Overexpression of ABCG5 and ABCG8 promotes biliary cholesterol secretion and reduces fractional absorption of dietary cholesterol. J Clin Invest. 2002;110(5):671-680.

15. Schuppan D, Kim YO. Evolving therapies for liver fibrosis. J Clin Invest. 2013;123(5):1887-1901.

16. Zhang Y, et al. E2F1 is a novel fibrogenic gene that regulates cholestatic liver fibrosis through the Egr-1/SHP/EID1 network. Hepatology. 2014;60(3):919-930.

17. Parker RA, et al. Bile acid and sterol metabolism with combined HMG-CoA reductase and PCSK9 suppression. J Lipid Res. 2013;54(9):2400-2409.

18. Fon Tacer K, Rozman D. Nonalcoholic Fatty liver disease: focus on lipoprotein and lipid deregulation. J Lipids. 2011;2011:783976. 
19. Arguello G, Balboa E, Arrese M, Zanlungo S. Recent insights on the role of cholesterol in non-alcoholic fatty liver disease. Biochim Biophys Acta. 2015;1852(9):1765-1778.

20. Ioannou GN. The Role of Cholesterol in the Pathogenesis of NASH. Trends Endocrinol Metab. 2016;27(2):84-95.

21. Musso G, Gambino R, Cassader M. Cholesterol metabolism and the pathogenesis of non-alcoholic steatohepatitis. Prog Lipid Res. 2013;52(1):175-191.

22. Poirier S, Mayer G. The biology of PCSK9 from the endoplasmic reticulum to lysosomes: new and emerging therapeutics to control low-density lipoprotein cholesterol. Drug Des Devel Ther. 2013;7:1135-1148.

23. Dubuc G, et al. Statins upregulate PCSK9, the gene encoding the proprotein convertase neural apoptosis-regulated convertase-1 implicated in familial hypercholesterolemia. Arterioscler Thromb Vasc Biol. 2004;24(8):1454-1459.

24. Shende VR, Wu M, Singh AB, Dong B, Kan CF, Liu J. Reduction of circulating PCSK9 and LDL-C levels by liver-specific knockdown of HNF1 $\alpha$ in normolipidemic mice. J Lipid Res. 2015;56(4):801-809.

25. Tabas I. Consequences of cellular cholesterol accumulation: basic concepts and physiological implications. J Clin Invest. 2002;110(7):905-911.

26. Giunzioni I, et al. Local effects of human PCSK9 on the atherosclerotic lesion. J Pathol. 2016;238(1):52-62.

27. Le May C, et al. Proprotein convertase subtilisin kexin type 9 null mice are protected from postprandial triglyceridemia. Arterioscler Thromb Vasc Biol. 2009;29(5):684-690.

28. Lalanne F, et al. Wild-type PCSK9 inhibits LDL clearance but does not affect apoB-containing lipoprotein production in mouse and cultured cells. J Lipid Res. 2005;46(6):1312-1319.

29. Shalem O, et al. Genome-scale CRISPR-Cas9 knockout screening in human cells. Science. 2014;343(6166):84-87.

30. Salmon P, Trono D. Production and titration of lentiviral vectors. Curr Protoc Neurosci. 2006; Chapter 4:Unit 4.21.

31. Benhamed F, et al. The lipogenic transcription factor ChREBP dissociates hepatic steatosis from insulin resistance in mice and humans. J Clin Invest. 2012;122(6):2176-2194.

32. Lopez-Mejia IC, et al. Antagonistic functions of LMNA isoforms in energy expenditure and lifespan. EMBO Rep. 2014;15(5):529-539.

33. Dikkers A, Freak de Boer J, Annema W, Groen AK, Tietge UJ. Scavenger receptor BI and ABCG5/G8 differentially impact biliary sterol secretion and reverse cholesterol transport in mice. Hepatology. 2013;58(1):293-303.

34. Denechaud PD, et al. ChREBP, but not LXRs, is required for the induction of glucose-regulated genes in mouse liver. $J$ Clin Invest. 2008;118(3):956-964.

35. Folch J, Lees M, Sloane Stanley GH. A simple method for the isolation and purification of total lipides from animal tissues. J Biol Chem. 1957;226(1):497-509. 